\title{
Unternehmenssteuerreform 2008
}

\begin{abstract}
Gewinner dervon der Bundesregierung geplanten Unternehmenssteuerreform 2008 sind vor allem ertragsstarke Unternehmen. Verschuldete und innovative Unternehmen mit Anlaufverlusten sowie der Mittelstand zählen hingegen zu den Verlierern. Zudem werden systemtragende Prinzipien der Einkommensbesteuerung verletzt. $\mathrm{Zu}$ diesem Ergebnis kommt eine Studie des ZEW und der Universität Mannheim (erschienen in „Der Betrieb“).
\end{abstract}

Reformszenarien zur Berechnung der Steuerbelastung in Deutschland

$$
\text { Reformoption } 1
$$

Reformoption 2

Reformoption 3

Änderungen auf Ebene der Kapitalgesellschaft

\section{Senkung des Tarifs der Körperschaftsteuer auf 12,5\%}

Reduzierung der Gewerbesteuermesszahl auf 4\%

kein Abzug der Gewerbesteuer als Betriebsausgabe

\begin{tabular}{c|c|c}
$\begin{array}{c}\text { 50\% Hinzurechnung } \\
\text { der Fremdkapital- } \\
\text { zinsen sowie des }\end{array}$ & $\begin{array}{c}100 \% \text { Hinzurechnung } \\
\text { der Fremdkapitalzinsen }\end{array}$ & $\begin{array}{c}\text { Beschränkung des jährlichen } \\
\text { Fremdkapitalzinsabzugs } \\
\text { auf } 60 \% \text { des Gewinns }\end{array}$ \\
\hline $\begin{array}{c}\text { Zinsanteils (25\%) der } \\
\text { Mieten, Pachten und }\end{array}$ & $\begin{array}{c}\text { vor Berücksichtigung der } \\
\text { Fremdkapitalzinsen/ }\end{array}$ \\
\hline $\begin{array}{c}\text { Lizenzgebühren zum } \\
\text { Gewinn vor Steuern }\end{array}$ & $\begin{array}{c}\text { unbeschränkter Abzug bis zu } \\
\text { einer Höhe von 1 Mio. Euro }\end{array}$
\end{tabular}

Änderungen auf Ebene der Anteilseigner
Abgeltungssteuer auf Zinsen, Dividenden und Veräußerungsgewinne in Höhe von $30 \%$ durchweg Entlastungen. Diese variieren allerdings in Abhängigkeit von der Gegenfinanzierung: Bei hälftiger Hinzurechnung der Zinsen (Option 1) liegt die durchschnittliche Entlastung bei 17 Prozent, bei einem vollständigen Abzugsverbot für Zinsen (Option 2) hingegen bei lediglich 12 Prozent. Wird der Abzug auf 60 Prozent des Gewinns begrenzt (Option 3), beträgt die Entlastung etwa 25 Prozent.

Die vorgesehenen Maßnahmen entlasten vor allem ertragsstarke und mit Eigenkapital finanzierte Unternehmen. Mit zunehmendem Verschuldungsgrad und einer schlechteren Ertragslage fällt die Entlastung dagegen deutlich geringer aus. Im Branchenvergleich können sich auch Mehrbelastungen ergeben, wie beispielsweise im Verkehrsgewerbe mit knapp 20 Prozent. Besonders innovative und damit auch riskante Unternehmen, die typischerweise Anlaufverluste haben, zählen zu den Verlierern der Reform.

Im internationalen Vergleich verbessert sich Deutschland im Länderranking

regierung die Eckpunkte der für das Jahr 2008 geplanten Unternehmensteuerreform beschlossen. Die Tarifbelastung der Gewinne für Kapitalgesellschaften soll auf knapp unter 30 Prozent sinken. Dazu soll der Körperschaftsteuersatz von 25 Prozent auf 12,5 Prozent halbiert und die Gewerbesteuermesszahl von 5 Prozent auf 4 Prozent reduziert werden. Gleichzeitig entfällt die Abzugsfähigkeit der Gewerbesteuer als Betriebsausgabe.

Zur Finanzierung der Einnahmeausfälle aus den Tarifsenkungen prüft derzeit die Bundesregierung drei Optionen, die in der Tabelle dargestellt sind. Mit Hilfe des am ZEW in Kooperation mit der
Universität Mannheim entwickelten „European Tax Analyzer“ wurden die Folgen der geplanten Maßnahmen für die effektive Steuerbelastung deutscher Kapitalgesellschaften abgeschätzt. Auf der Ebene des Unternehmens erfolgt dabei eine Senkung der Tarifbelastung mit Körperschaftsteuer einschließlich Solidaritätszuschlag und Gewerbesteuer von derzeit etwa 39 Prozent auf 30 Prozent. Als Gegenfinanzierungsmaßnahmen dienen jeweils die derzeit diskutierten drei Optionen.

Für ein typisches mittelständisches Unternehmen des verarbeitenden Gewerbes ergeben sich über einen Berechnungszeitraum von zehn Perioden zwar

\section{IN DIESER AUSGABE}

Unternehmenssteuerreform $2008 \ldots \ldots \ldots 1$ ZEW intern .................. 2 Ausgabenreformen in OECD-Staaten.................. 3 EU-Emissionshandel: Internationale Vernetzung bringt nur geringen Nutzen . . . 4 4 Unternehmen des IT- und Mediensektors nutzen E-Business am häufigsten ....... 5 Termine, Neuerscheinungen . . . . . . . . 6 Daten und Fakten $\ldots \ldots \ldots \ldots \ldots \ldots . \ldots$ Standpunkt ................. 8 
FORSCHUNGSERGEBNISSE

nur unwesentlich. Derzeit nimmt Deutschland unter zwölf Ländern den vorletzten Rang ein. Greifen die Abzugsbeschränkungen für Fremdkapitalzinsen, verbessern sich deutsche Kapitalgesellschaften lediglich um zwei Positionen auf den neunten Rang. Eine spürbare Verbesserung der Standortattraktivität ist somit nicht zu erwarten. Wollte man dies erreichen, müssten die vorgesehenen Abzugsbeschränkungen weniger drastisch ausfallen.

Weiterhin plant die Bundesregierung, im Zuge der Unternehmensteuerreform aus Gründen der Steuervereinfachung und zur Verbesserung der Finanzierungsneutralität der Besteuerung auf private Kapitalerträge eine Abgeltungssteuer in Höhe von 30 Prozent einzuführen. Für Dividenden und Veräußerungsgewinne entfällt das bisher geltende Halbeinkünfteverfahren.

Die geplante Abgeltungssteuer führt in Kombination mit den auf Unternehmensebene vorgesehenen Zinsabzugsbeschränkungen zu einer Doppelbesteuerung der in Unternehmen erwirtschafteten Erträge. Damit ist tendenziell eine steuerliche Mehrbelastung mittelständischer Unternehmen verbunden, bei denen die Gesamtsteuerbelastung auf Unternehmensebene und auf Ebene der Gesellschafter relevant ist. In Abhängigkeit vom Ausmaß der Abzugsbeschränkung ergeben sich Mehrbelastungen zwischen 1,7 und 4,15 Prozent. Hiermit verschlechtert sich für den Mittelstand die steuerliche Wettbewerbsfähigkeit gegenüber im Ausland ansässigen Konkurrenten.

Prof.Dr.Christoph Spengel, spengel@zew.de Timo Reister, reister@zew.de

\section{ZEW INTERN}

\section{Sabine Jokisch erhält DIA-Zukunftspreis}

Dr. Sabine Jokisch, Wissenschaftlerin im ZEW-Forschungsbereich Umwelt- und Ressourcenökonomik, Umweltmanagement, ist vom Deutschen Institut für Altersvorsorge für ihre Dissertation „The Developed World's Demographic Transition - Implications for Fiscal Policy and the International Macroeconomy“ mit dem DIA-Zukunftspreis 2006 ausgezeichnet worden.

In fast allen Industrieländern steigt der Anteil der Alten in der Bevölkerung enorm. Die Arbeit untersucht die makroökonomischen und finanzpolitischen Wirkungen der alternden Bevölkerungen in den USA, der EU und Japan innerhalb eines dynamischen Gleichgewichtsmodells mit überlappenden Generationen. Die Simulationsergebnisse zeigen, dass der demographische Wandel in allen drei Regionen zu einem dramatischen Anstieg der Sozialversicherungsbeiträge führt. Diese Entwicklungen schränken die künftige Kapitalakkumulation ein, senken die Löhne und erhöhen den Realzinssatz. Die Studie simuliert verschiedene Reformvorschläge, um zu untersuchen, ob diese die demographischen Belastungen lindern können. Änderungen in der Fertilitäts- und Mortalitätsrate haben dabei einen geringen

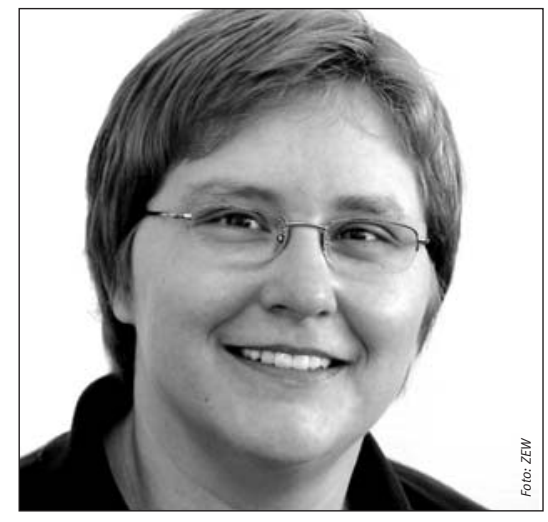

Dr. Sabine Jokisch

Einfluss auf die zukünftige wirtschaftliche Entwicklung. Gezielte Einwanderung könnte das demograpische Problem nur lösen, wenn es den Industrienationen gelänge, viele gut ausgebildete Fachkräfte zu gewinnen. In weiteren Simulationen zeigt sich, dass eine grundlegende Rentenreform, wie etwa die volle Privatisierung der Rentenkassen, ein vielversprechendes Mittel ist.

\section{Johannes Rincke bei IIPF-Tagung ausgezeichnet}

Johannes Rincke, LMU München und bis März 2006 Mitarbeiter am ZEW, hat auf der 62. Jahrestagung des International Institute for Public Finance den Peggy and Richard Musgrave Preis für das beste Papier eines Nachwuchswissen- schaftlers gewonnen. Prämiert wurde sein Papier „Yardstick competition and public sector innovation“, das im Rahmen seiner am ZEW entstandenen Dissertation erarbeitet wurde. Das Papier untersucht die Rolle des Wettbewerbs zwischen lokalen Gebietskörperschaften bei der Bestimmung der Innovationsbereitschaft des öffentlichen Sek-

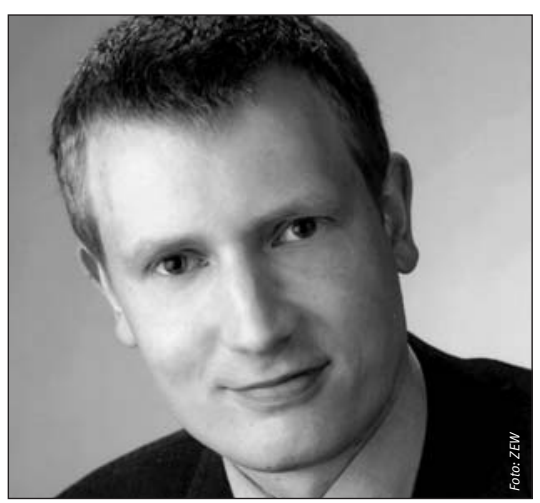

Johannes Rincke

tors. In einem theoretischen Teil zeigt er, wie politische Entscheidungsträger Innovationen strategisch zur Stärkung ihrer Reputation einsetzen können. Anhand von Politikinnovationen in USamerikanischen Gebietskörperschaften weist die Arbeit schließlich nach, dass wesentliche Implikationen der Theorie im Hinblick auf die räumliche Verteilung von Politikinnovationen empirisch gestützt werden. 


\section{Ausgabenreformen in OECD-Staaten}

Die Ausgabenseite öffentlicher Haushalte gewinnt durch die Notwendigkeit zur Konsolidierung und im Hinblick auf die Qualität der öffentlichen Finanzen zunehmend an Aufmerksamkeit in der wirtschaftspolitischen Debatte. Hohe Staats- und Schuldenquoten sowie der Globalisierungsdruck hatten in zahlreichen OECD Staaten bereits erhebliche budgetäre Anpassungen zur Folge. Eine gemeinsame Studie von Ökonomen des ZEW und der Europäischen Zentralbank untersucht erfolgreiche ausgabenseitige Haushaltskonsolidierungen dervergangenen Jahrzehnte und kommt zu dem Schluss, dass diese im Rahmen umfangreicher Reformpakete stattfanden.

Die Notwendigkeit einer nachhaltigen Konsolidierung der öffentlichen Haushalte bestimmt seit geraumer Zeit die wirtschaftspolitische Diskussion in Deutschland. Insbesondere das wiederholte Verfehlen der Maastrichter Verschuldungskriterien hat den Druck auf die finanzpolitischen Akteure von Bund und Ländern erhöht. Die Bundesregierung erklärte bei ihrer Regierungsübernahme, das 3 v.H.-Defizitkriterium ab dem Jahr 2007 wieder erfüllen zu wollen. Dazu beschloss sie unter anderem eine Erhöhung des allgemeinen Mehrwertsteuersatzes um drei Prozentpunkte.

Zahlreiche empirische Studien untersuchen die Frage nach den Erfolgsaussichten verschiedener fiskalischer Konsolidierungsstrategien. Sie kommen überwiegend zu dem Ergebnis, dass fiskalische Konsolidierung vor allem dann erfolgreich ist, wenn staatliche Ausgaben, insbesondere Personal- und Transferausgaben, gekürzt werden. In den vergangenen Jahrzehnten haben zahlreiche OECD Länder ihre Staatsquoten (Staatsausgaben bezogen auf das Bruttoinlandsprodukt) deutlich gesenkt. Die Studie von ZEW und EZB (ZEW Discussion Paper No. 06-050) identifiziert mit Hilfe eines Klassifizierungsschemas insgesamt elf „ambitionierte“ siebenjährige Reformphasen seit 1980. Diese wurden im Rahmen detaillierter Fallstudien in Bezug auf das makroökonomische Umfeld, den Umfang und die Zusammensetzung der Ausgabensenkung und wichtige institutionelle Komponenten der Reformbemühungen analysiert.

Es zeigt sich, dass nachhaltige Ausgabenreformen im Kontext einer umfassenden Reformagenda durchgeführt wurden. Hauptaugenmerk lag bei den Ausgabenkürzungen typischerweise auf den konsumtiven Staatsausgaben sowie den Transfers und Subventionen. Durchschnittlich entfielen mehr als 80 Prozent

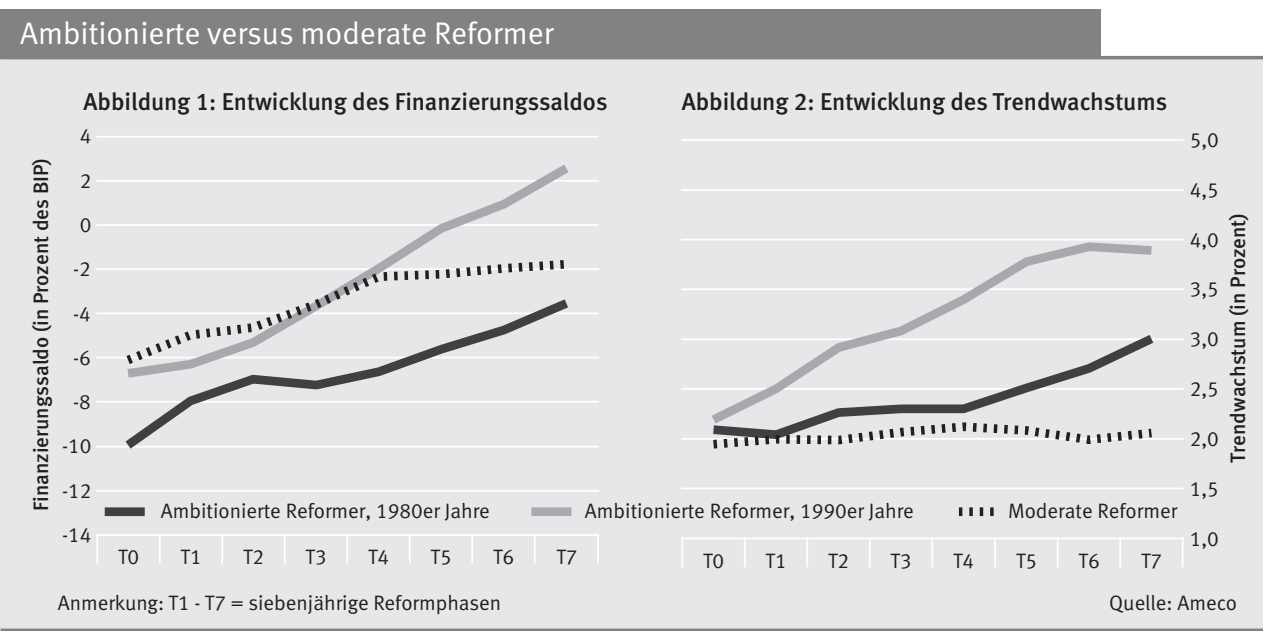

des Rückgangs der (Primär-)Ausgabenquote auf diese Kategorien. Flankiert wurde die ausgabenseitige Konsolidierung häufig durch Liberalisierungs- und Privatisierungsprozesse auf Arbeits-, Güter- und Dienstleistungsmärkten sowie durch Steuerreformen und Stärkung der Fiskalinstitutionen.

\section{Einbeziehung der Ausgabenseite}

Abbildung 1 verdeutlicht die durchschnittliche Entwicklung des Finanzierungssaldos für die Gruppe der „ambitionierten“ Reformer im Vergleich zur Entwicklung in „moderaten“ Reformphasen, die durch geringere Ausgabensenkungen charakterisiert waren. Unabhängig vom Zeitpunkt der Reform hat sich bei „ambitionierten“ Reformern - verglichen mit der Kontrollgruppe - der Fi- nanzierungssaldo deutlicher verbessert. Durchschnittlich dienten etwa zwei Drittel der Ausgabenkürzungen zur Verringerung des Defizits, während das verbleibende Drittel für Steuersenkungen herangezogen wurde. Trotz erheblicher Anpassungen auf der Ausgabenseite ist bei „ambitionierten“ Reformern eine günstigere Wachstumsentwicklung festzustellen (Abbildung 2). Dies legt den Schluss nahe, dass positive Erwartungseffekte durch glaubwürdige Konsolidierungsund Reformstrategien zu einem Wachstumsaufschwung beitragen können. 


\section{EU-Emissionshandel: Internationale Vernetzung bringt nur geringen Nutzen}

Das europäische Emissionshandelssystem soll künftig international vernetzt werden. Staaten wie Kanada, Japan oder Australien planen ähnliche Handelssysteme auf Unternehmensebene, die mit dem europäischen System verknüpft werden könnten. Eine aktuelle Studie des ZEW zeigt, dass eine solche Vernetzung unter den derzeitigen Bedingungen nur geringen ökonomischen Nutzen brächte. Die flexiblen Mechanismen des Kioto-Protokolls dagegen erhöhen die Kosteneffizienz der Klimapolitik und steigern so die Attraktivität einer internationalen Vernetzung.

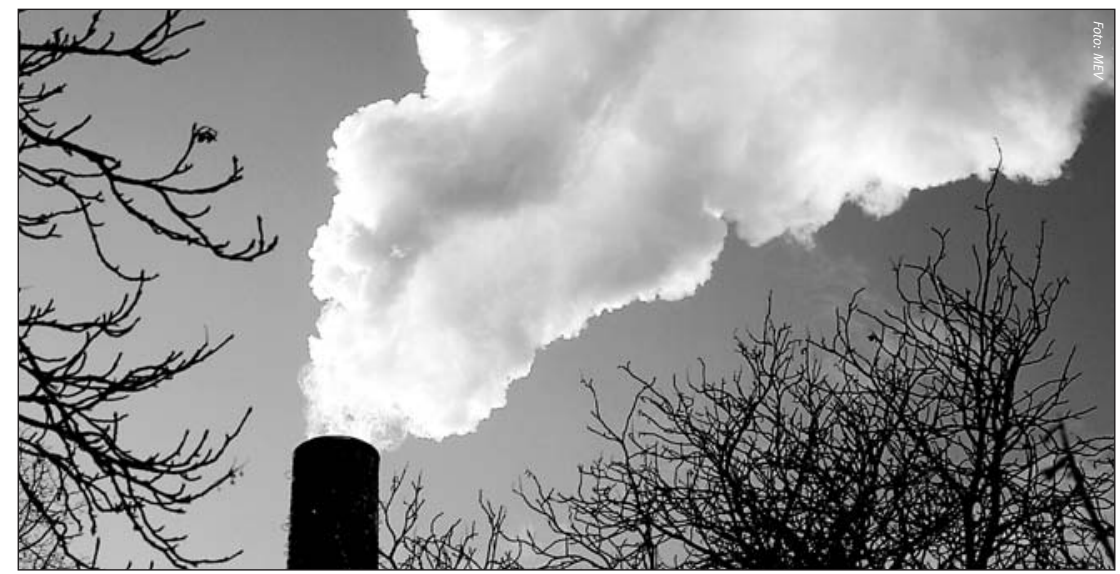

Auf der zwölften Weltklimakonferenz in Nairobi treffen ab 6. November 2006 die Staaten der Vereinten Nationen zusammen, um die Zukunft des Klimaschutzes zu gestalten. Auf der Tagesordnung steht neben der Überarbeitung des Kioto-Protokolls vor allem die Verhandlung eines neuen Abkommens ab dem Jahr 2012, denn das Protokoll gilt vorerst nur bis zu diesem Zeitpunkt.

Um die Verpflichtungen der EU aus dem Kioto-Protokoll, Treibhausgasemissionen um durchschnittlich acht Prozent gegenüber 1990 zu senken, kostengünstig zu erfüllen, hat die europäische Kommission das EU-Emissionshandelssystem eingeführt. Das seit dem Jahr 2005 aktive System ermöglicht es energieintensiven Unternehmen der Mitgliedsstaaten, untereinander Verschmutzungsrechte zu handeln. Emissionshandel soll in Zukunft jedoch nicht auf Europa beschränkt bleiben: Kanada, Japan oder Australien planen ähnliche Handelssysteme auf Firmenebene, die künftig mit dem europäischen System verknüpft werden könnten. Die EU-Kommission hat Interesse an dieser Strategie, da hierdurch der internationale Markt für Emissionsrechte wachsen würde.

Eine neue Studie des ZEW (Discussion Paper No. 06-058) zeigt anhand eines ökonomischen Simulationsmodells, dass eine internationale Vernetzung des EUEmissionshandelssystems nur leicht positive Effekte hat. Das EU-System reguliert derzeit nur die energieintensiven Branchen der europäischen Volkswirtschaften. Zudem wurden den teilnehmenden Firmen relativ großzügig Emissionsrechte zugeteilt. Da das EU-System als Vorlage für die geplanten außereuropäischen Systeme dienen soll, profitieren die energieintensiven Branchen der EU nur wenig vom erweiterten Emissionshandel, da sie aufgrund der großzügigen Zuteilung geringe Anpassungskosten haben, die durch erweiterten Emissionshandel nur noch leicht sinken können. Um die nationalen Reduktionsziele zu erreichen, muss daher der Großteil der Emissionsvermeidung von Sekto- ren der europäischen Volkswirtschaften durchgeführt werden, die nicht dem Emissionshandel unterliegen (wie etwa dem Verkehrs- oder Haushaltssektor). Diese Branchen tragen auch die ökonomische Hauptlast, da hier die Kosten der Emissionsvermeidung vergleichsweise hoch sind und sie nicht von den erweiterten Handelsmöglichkeiten profitieren.

Die „flexiblen Mechanismen“ des Kioto-Protokolls könnten hier Abhilfe schaffen. So ermöglicht das Protokoll ebenfalls internationalen Emissionshandel, allerdings zwischen Staaten. Dieser findet ab Inkrafttreten des Protokolls im Jahr 2008 parallel zum Emissionshandel auf Unternehmensebene statt. Staaten können zudem in „saubere“Technologien, etwa erneuerbare Energieträger, in Entwicklungsländern investieren und sich die entsprechenden Emissionsminderungen gutschreiben lassen (Clean Development Mechanism, CDM).

Die ZEW-Studie zeigt, dass die Ineffizienzen - aufgrund der Beschränkung auf energieintensive Branchen und der großzügigen Zuteilung - der gegenwärtigen und geplanten Emissionshandelssysteme durch parallelen Staatenhandel und CDM unter einem Klimaschutzabkommen über das Jahr 2012 hinaus abgefedert werden können. So werden gerade die Wirtschaftsbereiche mit geringer Energieintensität, die nicht dem Emissionshandel unterliegen, stark kompensiert, indem die Regierung über das Kioto-Protokoll zusätzliche Emissionsrechte importiert und auf diese Weise die nationalen Reduktionsziele erfüllt. Eine zukünftige Klimapolitik könnte zudem Emissionshandel zwischen Unternehmen und Staaten ermöglichen. In dieser Situation ist der ökonomische Nutzen einer internationalen Vernetzung des EU-Systems am größten, da hierdurch die höchstmögliche Flexibilität im Emissionshandel geschaffen wird.

Niels Anger, anger@zew.de 


\section{Unternehmen des IT- und Mediensektors nutzen E-Business am häufigsten}

Fast 63 Prozent der baden-württembergischen Unternehmen setzen computergestützte Anwendungen zur Vernetzung und Automatisierung ihrer Geschäftsprozesse (E-Business) ein. Allerdings gibt es große Unterschiede zwischen den Branchen. Dies zeigt die dritte repräsentative FAZIT-Unternehmensbefragung, die das ZEW im Juni und Juli 2006 in Baden-Württemberg durchgeführt hat.

An der Befragung beteiligten sich mehr als 700 Unternehmen aus dem ITund Mediensektor, dem verarbeitenden Gewerbe und dem Bank- und Versicherungsgewerbe sowie Verkehrsdienstleister und technische Dienstleister. Das „Forschungsprojekt für aktuelle und zukunftsorientierte IT und Medientechnologien und deren Nutzung in Baden-Württemberg“ (FAZIT) ist ein durch das Land gefördertes gemeinnütziges Forschungsprojekt (www.fazit-forschung.de).

Die Unternehmen des IT- und Mediensektors nutzen mit fast 68 Prozent E-Business am häufigsten. Ebenfalls auf große Resonanz stößt E-Business bei den Verkehrsdienstleistern (62 Prozent). Bei Banken und Versicherungen sind E-Business-Lösungen bisher am geringsten verbreitet. Nur knapp 49 Prozent der Unternehmen dieser Branche, die auch Kredit- und Versicherungsmakler umfasst, setzen auf computergestützte Systeme zur Vernetzung und Automatisierung ihrer Geschäftsprozesse. Bei den Unternehmen des verarbeitenden Gewerbes und den technischen Dienstleistern liegt dieser Anteil bei jeweils knapp 59 Prozent.

E-Business-Lösungen kommen verstärkt in größeren Unternehmen zum Einsatz. Die Nutzungsrate bei Großunternehmen mit mindestens $250 \mathrm{Be}$ schäftigten erreicht 91 Prozent, wohingegen nur knapp 55 Prozent der kleinen Unternehmen mit bis zu vier Beschäftigten E-Business-Lösungen einsetzen.
Haupteinsatzbereich von E-BusinessLösungen ist die Buchhaltung (siehe Abbildung). Insgesamt die Hälfte aller Unternehmen nutzt in diesem Unternehmensbereich die Vorteile von E-Business. Beim Einkauf setzen noch gut 37 Prozent der Unternehmen auf E-Business-Lösungen, beispielsweise in Form von Online-Shops, Online-Marktplätzen oder Auktionen. In der Kundenbetreuung verwenden 27 Prozent der Unternehmen E-Business-Lösungen. Bisher wenig Verbreitung findet E-Business dagegen in der Produktion (9 Prozent) oder in unternehmensübergreifenden Systemen (Supply Chain Management) (4 Prozent). großen Wert auf Kompatibilität mit anderen oder bestehenden Lösungen, fast ebenso viele auf die Vernetzbarkeit mit anderen Lösungen (78 Prozent). Standardisierte Lösungen sind für mehr als 70 Prozent der Unternehmen wichtig.

\section{Konsequenzen des Einsatzes von E-Business}

Die Folgen des Einsatzes von E-Business-Lösungen im Unternehmen sind vielfältig. So verzeichnen 57 Prozent der Unternehmen eine Beschleunigung ihrer Geschäftsprozesse und 46 Prozent qualitativ verbesserte Unternehmenspro-

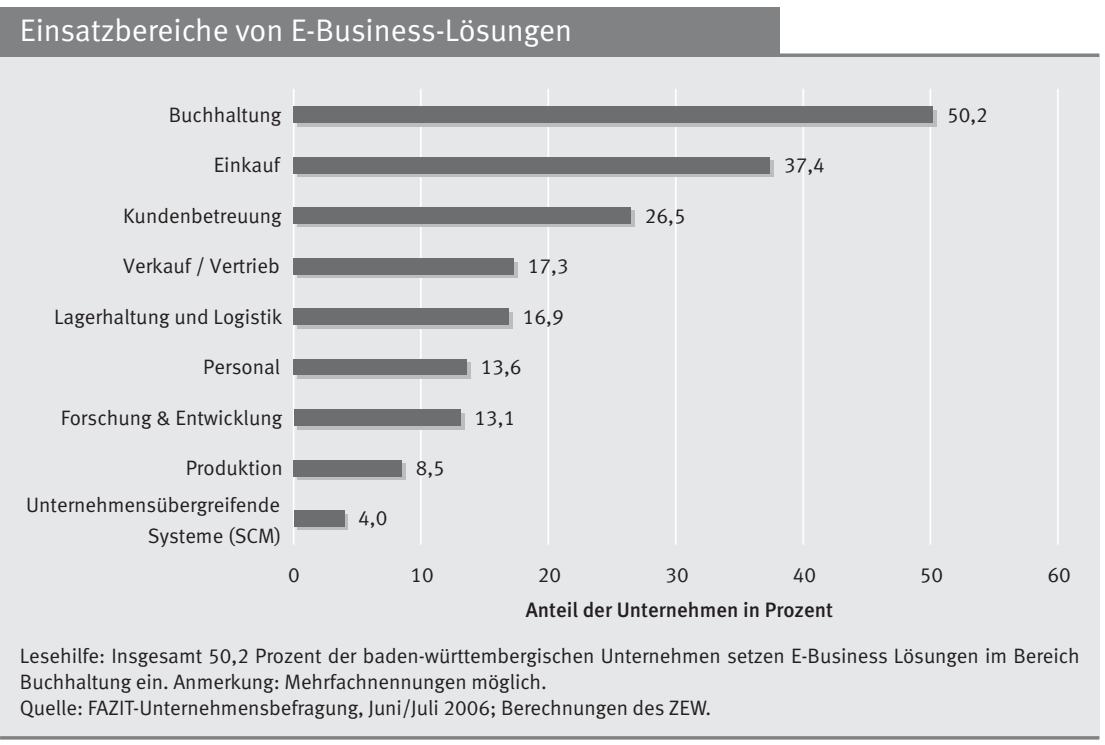

Bei der Wahl der E-Business-Lösung spielen die damit einhergehenden Kosten eine herausragende Rolle. Annähernd 89 Prozent der Unternehmen, die E-Business-Lösungen verwenden, bezeichnen niedrige Kosten als das wichtigste Auswahlkriterium für ihre Entscheidung. Weitere bedeutende Entscheidungskriterien beziehen sich auf das Zusammenspiel der E-Business-Lösung mit der bereits vorhandenen Softwareumgebung im Unternehmen. Mehr als 84 Prozent der Unternehmen legen zesse. Bei insgesamt 43 Prozent führt die E-Business-Implementierung zu sinkenden Kosten. Auch auf die Informationsflüsse wirkt sich der E-BusinessEinsatz positiv aus: Gut 35 Prozent der Unternehmen stellen einen verbesserten Informationsaustausch mit Kunden und Zulieferern fest, und bei fast 32 Prozent funktioniert der Informationsaustausch innerhalb des Unternehmens beziehungsweise zwischen einzelnen Unternehmensteilen nun besser.

JörgOhnemus, ohnemus@zew.de 
ZEW DISCUSSION PAPERS

No. 06-053, Miriam Beblo, Stefan Bender, Elke Wolf: The Wage Effects of Entering Motherhood - A Within-firm Matching Approach.

No. 06-054, Reinhard Hujer, Stephan Lothar Thomsen: Wirksamkeit von Arbeitsbeschaffungsmaßnahmen in Deutschland: Empirische Befunde mikroökonometrischer Analysen.

No. 06-055, Dirk Czarnitzki, Andrew A. Toole: Business R\&D and the Interplay of R\&D Subsidies and Market Uncertainty.

No. 06-056, Dirk Czarnitzki, Andrew A. Toole: Patent Protection, Market Uncertainty, and R\&D Investment.

No. 06-057, Peter Westerheide: Cointegration of Real Estate Stocks and REITs with Common Stocks, Bonds and Consumer Price Inflation - an International Comparison.

No. 06-058, Niels Anger: Emission Trading Beyond Europe: Linking Schemes in a Post-Kyoto World.

No. 06-059, Birgit Aschhoff, Tobias Schmidt: Empirical Evidence on the Success of R\&D Co-operation - Happy Together?

No. 06-060, Andreas Ammermüller: Pupil-Teacher Gender Interaction Effects on Scholastic Outcomes in England and the USA.

No. 06-061, Melanie Arntz, Ralf Wilke, Henrik Winterhager: Regionenmatching im Rahmen der Evaluation der Experimentierklausel des § 6c SGB II: Methodische Vorgehensweise und Ergebnisse.

No. 06-062, Melanie Arntz: What Attracts Human Capital? Understanding the Skill Composition of Interregional Job Matches in Germany.

No. 06-063, Kris Aerts, Tobias Schmidt: Two for the Price of One? On Additionality Effects of R\&D Subsidies: A Comparison Between Flanders and Germany.

No. 06-064, Alexander Spermann: Basic Income Reform in Germany: Better Gradualism than Cold Turkey.

No. 06-065, Reinhard Hujer, Stephan Lothar Thomsen, Christopher Zeiss: The Effects of Short-Term Training Measures on the Individual Unemployment Duration in West Germany.

No. 06-066, Bernd Fitzenberger, Karsten Kohn, Qingwei Wang: The Erosion of Union Membership in Germany: Determinants, Densities, Decompositions.

\section{ZEW SCHRIFTENREIHEN}

Georg Bühler

Verkehrsmittelwahl im Güter-
verkehr. Eine Analyse ordnungs-
und preispolitischer Maßnahmen

Das Buch erklärt auf der Grundlage einer Breitenbefragung das Entscheidungsverhalten bezüglich der Verkehrsmittelwahl im Güterverkehr.

Da aufgrund einer stark steigenden Transportnachfrage im Güterverkehr externe Kosten in erheblichem Umfang verursacht werden, muss die Verkehrspolitik gezielt Maßnahmen ergreifen, um diese Mehrnachfrage möglichst umweltverträglich zu gestalten. Deshalb streben die EU und die Bundesregierung an, den Verkehr von der Straße auf alternative Verkehrsträger zu verlagern. Diese Arbeit analysiert, von welchen Bestimmungsfaktoren die Verkehrsmittelwahl der Spediteure abhängt, um daran anschlieBend den Effekt bestimmter Regulierungen zu ermitteln. So wird etwa die Auswirkung der Lkw-Maut auf die Verkehrsmittelwahl untersucht.

ZEW Schriftenreihe Umwelt- und Ressourcenökonomie, Physica-Verlag, 2006, ISBN 3-7908-1753-8

\section{EXPERTENSEMINARE}

25.10.2006 Training for IFRS: Impairment Test und Goodwill Bilanzierung nach IFRS 3 und IAS 36 (in Zusammenarbeit mit Ernst\&Young)

25.10.2006 Allgemeines Gleichbehandlungsgesetz (AGG)

26.10.2006 Financial Instruments nach IFRS in der Praxis (IAS 32, 39 und IFRS 7) (in Zusammenarbeit mit Ernst\&Young)

7.11.2006 Bilanzpolitik in mittelständischen Unternehmen Analyse und Gestaltung von Jahresabschlüssen

8.11.2006 Befragungen kompetent gestalten und durchführen

9.11.2006 Volkswirtschaftliche Daten für Führungskräfte

15.11.2006 Training for IFRS: Vorratsbewertung (in Zusammenarbeit mit Ernst\&Young)

16.11.2006 Internationaler Mittelstandstag

20.11.2006 Ökonometrie Basistechniken I-Regressionsanalyse

21.11.2006 Ökonometrie Basistechniken II - Zeitreihenmodelle

28.11.2006 Mezzanine Finanzinstrumente Bilanzielle und steuerrechtliche Behandlung

ZEW-Expertenseminare im Internet: www.zew.de $\rightarrow$ Weiterbildung Information: Vera Pauli, Telefon 0621/1235-240, E-Mail pauli@zew.de

\section{ZEW DOKUMENTATION}

Dokumentation Nr. 06-05

Innovationsbarrieren und internationale Standortmobilität

Eine Studie im Auftrag der IG BCE, Chemieverbände Rheinland-Pfalz und der BASF Aktiengesellschaft

Autoren des ZEW: Thiess Büttner (jetzt LMU München), Thomas Cleff, Jürgen Egeln, Georg Licht, Georg Metzger, Michael Overesch, Christian Rammer Autoren des DIW: Heike Belitz, Dietmar Edler, Hella Engerer, Ingo Geishecker, Mechthild Schrooten, Harald Trabold, Axel Werwatz, Christian Wey

Die Dokumentation ist im Internet verfügbar und kann von der Webseite des ZEW heruntergeladen werden: http://www.zew.de/de/publikationen/ publikation.php3?action=detail\&art= $13 \& n r=3072$ 


\section{ZEW-Finanzmarkttest im September 2006}

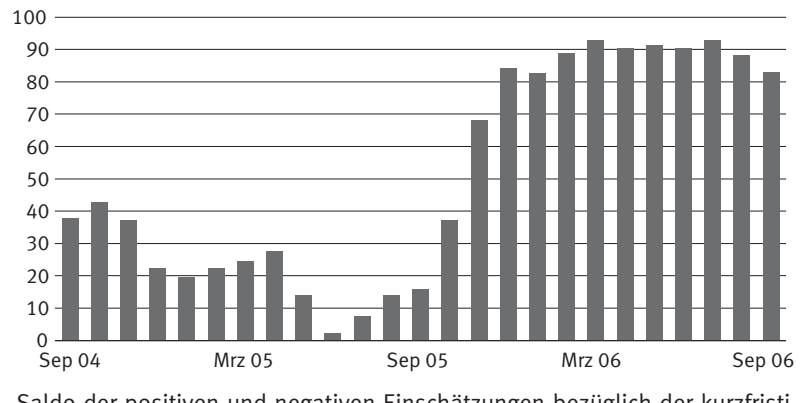

Saldo der positiven und negativen Einschätzungen bezüglich der kurzfristigen Zinsen im Euroraum in sechs Monaten.

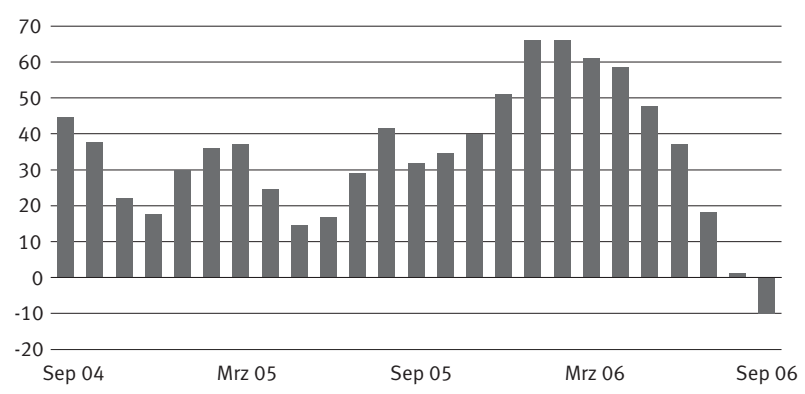

Saldo der positiven und negativen Einschätzungen bezüglich der Konjunktursituation im Euroraum in sechs Monaten.

\section{EZB: Zinserhöhungserwartungen gehen zurück}

Ein gesunkener Ölpreis dürfte den Inflationsdruck im Euroraum verringert haben. Im Zuge dessen sinken die Zinserhöhungserwartungen der Analysten geringfügig. Die Möglichkeit inflationstreibender Lohnabschlüsse in Folge der deutschen Mehrwertsteuererhöhung oder ein erneuter Ölpreisanstieg lassen jedoch eine Fortsetzung der Zinserhöhungspolitik erwarten. Diese Erwartungen haben in der jüngsten geldpolitischen Sitzung ihre Bestätigung gefunden - der geldpolitische Rat hat einen weiteren Zinsschritt auf nunmehr 3,25 Prozent vorgenommen. Sandra Schmidt, s.schmidt@zew.de

\section{Euroraum: Nachlassendes Wachstum erwartet}

Die ZEW-Konjunkturerwartungen für den Euroraum sind im September erneut gefallen. Der Saldo aus Optimisten und Pessimisten bezüglich der Konjunktursituation in sechs Monaten sinkt um 11,5 auf aktuell -10,2 Punkte. Dies deutet auf eine Abschwächung des Wachstums in der Eurozone hin. Ein Grund dafür ist, dass die Nachfrage aus den USA nachlassen dürfte, des weiteren wird befürchtet, dass die Erhöhung der Mehrwertsteuer in Deutschland und die Zinserhöhungen durch die EZB die inländische Nachfrage dämpfen könnten.

Sandra Schmidt, s.schmidt@zew.de

\section{Experten erwarten steigende Preise im Straßengüterverkehr}

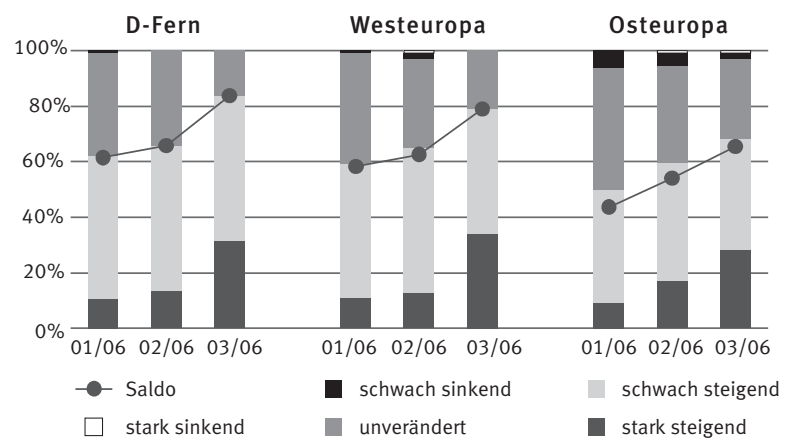

Quelle: ZEW

Die Experten des TransportmarktBarometers von ProgTrans/ ZEW erwarten für das kommende Halbjahr steigende Transportpreise. Diese werden neben den hohen (Treibstoff-)Kosten vor allem vom starken Mengenwachstum getrieben. Der Kostendruck osteuropäischer Wettbewerber kann diese Preissteigerungen nur wenig abfedern. Daher erwarten selbst in Verkehrsrichtung Osteuropa rund 70 Prozent der Experten steigende Preise, ansonsten gehen bis zu 85 Prozent von Preissteigerungen aus. Etwa 30 Prozent rechnen im nationalen Fern- und im internationalen Straßengüterverkehr sogar mit Preiserhöhungen von mehr als drei Prozent.

Dr.GeorgBühler, buehler@zew.de

\section{Kredit- und Versicherungsgewerbe: Ausweitung der Innovationsbeteiligung wahrscheinlich}

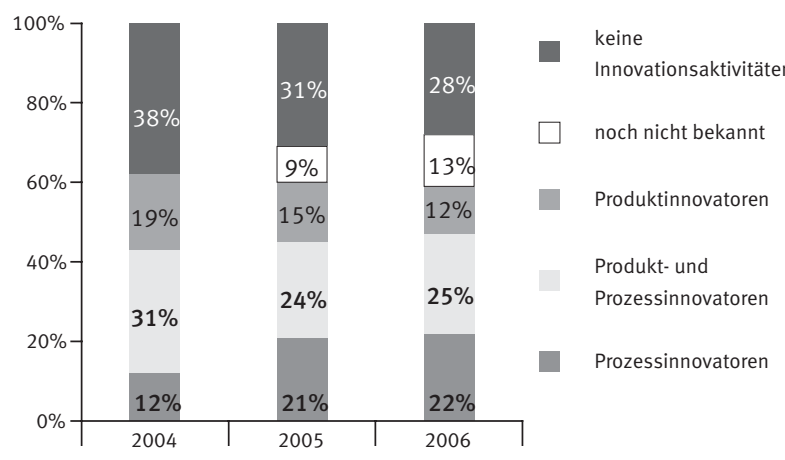

Quelle: ZEW

63 Prozent der Unternehmen des Kredit- und Versicherungsgewerbes waren im Jahr 2004 innovativ tätig. Für 2006 rechneten zum Befragungszeitpunkt (Mitte 2005) bereits 59 Prozent der Unternehmen fest mit Innovationsaktivitäten. Da sich gleichzeitig noch 13 Prozent der Unternehmen bezüglich ihrer künftigen Innovationsaktivitäten unsicher waren, ist ein Anstieg der Innovationsbeteiligung im Jahr 2006 gegenüber 2004 wahrscheinlich. Sowohl 2005 als auch 2006 wollen die Unternehmen im Kredit- und Versicherungsgewerbe verstärkt Prozessinnovationen einführen. Dahinter stehen häufig Bemühungen, die Kosten zu senken. Heide Löhlein, loehlein@zew.de 


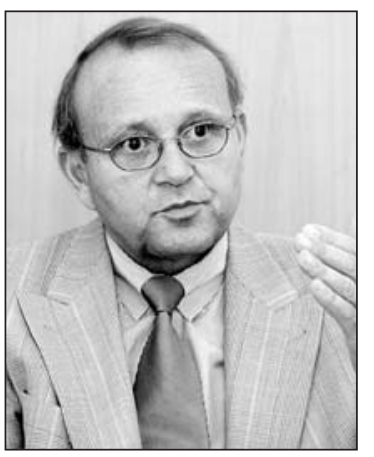

Kombilohn

Die Bundesregierung will bekanntlich die Einführung eines Kombilohns prüfen und hat in diesem Zusammenhang den Sachverständigenrat zur Begutachtung der gesamtwirtschaftlichen Entwicklung ersucht, ein Gutachten zu erstellen. Dem ist der Rat mit seiner Expertise „Arbeitslosengeld II reformieren: Ein zielgerichtetes Kombilohnmodell“ nachgekommen. Die Resonanz auf diesen Vorschlag war erfreulicherweise hoch, bedauerlicherweise aber zum Teil durch mehr als unsachliche Kritik gekennzeichnet. Reflexartig wurde - schon bevor die Einzelheiten des Modells bekannt waren - unterstellt, der Sachverständigenrat wolle den Empfängern des Arbeitslosengelds II das sozialstaatlich gebotene Existenzminimum beschneiden.

Davon kann überhaupt keine Rede sein. Jeder der Betroffenen kann trotz der vorgeschlagenen Senkung des Regelsatzes des Arbeitslosengelds II den vollen Betrag erhalten, jedoch muss er dafür eine Gegenleistung erbringen, und zwar in Form einer intensiven Suche nach einem Arbeitsplatz auf dem ersten Arbeitsmarkt oder hilfsweise durch Aufnahme einer Arbeit in einer Arbeitsgelegenheit. Es kann nicht deutlich genug darauf aufmerksam gemacht werden, dass das Arbeitslosengeld II keine Versicherungsleistung darstellt, sondern eine Fürsorgeleistung an hilfsbedürftige Personen. Dann aber hat die Gesellschaft das Recht, eine Gegenleistung der beschriebenen Art zu fordern. Das entspricht im übrigen der bestehenden Gesetzeslage im Sozialgesetzbuch, und die derzeitigen Regelungen sind eigentlich viel strenger als der Vorschlag des Sachverständigenrats. Denn bei Ablehnung von Arbeitsplatzangeboten kann bereits jetzt das Arbeitslosengeld II wesentlich stärker gekürzt werden als die 30 v.H., die das Modell des Sachverständigenrats enthält. Natürlich steht es dem Gesetzgeber frei, über die Kürzung in Höhe von 30 v.H. und damit über die Anregung des Sachverständigenrats hinauszugehen. Ein entscheidender Unterschied zur bestehenden Praxis ist die Umkehrung der Beweislast. Der Arbeitslose muss selbst aktiv werden, um das bisherige Leistungsniveau zu erreichen. Das Modell des Sachver- ständigenrats behandelt deshalb Arbeitslose gerade nicht als „Drückeberger“ oder „Faulenzer“, was sie in ihrer großen Mehrheit auch nicht sind.

Wieso knüpft das Modell des Rats am Arbeitslosengeld II an? Erstens besitzt das Arbeitslosengeld II bereits den Charakter eines Kombilohns, weil beispielsweise bei niedrigen $\mathrm{Er}$ werbseinkommen ein ergänzender Bezug von Arbeitslosengeld II, also eine Lohnsubvention, gewährt werden kann. Zweitens ist die Zielgruppenorientierung vergleichsweise hoch. Rund drei Viertel der arbeitslosen Geringqualifizierten und der Langzeitarbeitslosen - dies sind die Problemgruppen auf dem Arbeitsmarkt - können einen Anspruch auf Arbeitslosengeld II geltend machen. Drittens erfolgt im Rahmen des Arbeitslosengelds II eine Bedürftigkeitsprüfung, die ohnehin Voraussetzung für die Inanspruchnahme eines Kombilohns darstellen sollte.

Die Arbeitsgelegenheiten sind die ordnungspolitische Kröte, die man schlucken muss, denn anders lässt sich die Attraktivität regulärer Beschäftigung bei gleichzeitiger Wahrung des staatlich garantierten Mindesteinkommens nicht steigern. Es wäre blauäugig, die Gefahr von Verdrängungseffekten zu leugnen. Aber Übertreibungen sind ebenso verfehlt, und die Anzahl der Arbeitsgelegenheiten wird bei voller Wirksamkeit des Sachverständigenratsmodells niedriger sein als derzeit.

Nach den Berechnungen des Sachverständigenrats wird sich der positive Beschäftigungseffekt auf dem ersten Arbeitsmarkt auf etwa 350 Tausend Personen belaufen. Ein Kombilohn ist mithin kein Königsweg zur Bekämpfung der Arbeitslosigkeit. Er macht Reformen auf dem Arbeitsmarkt nicht entbehrlich und entlässt die Tarifvertragsparteien keineswegs aus ihrer Verantwortung. Die Lohnpolitik muss eine weitere Spreizung der qualifikatorischen Lohnstruktur insbesondere im Niedriglohnbereich vornehmen, das Mindesteinkommen bleibt gleichwohl gesichert.

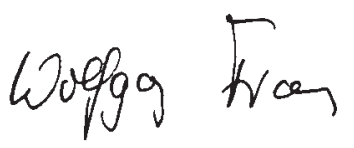

